Review

\title{
A perspective profile of ADCY1 in cAMP signaling with drug-resistance in lung cancer
}

\author{
Ting Zou ${ }^{1,2,3^{*}}$, Junyan Liu ${ }^{*}$, Li She5,6, Juan Chen7, Tao Zhu², Jiye Yin², Xi Li², Xiangping Li², Honghao Zhou ${ }^{2}$, \\ Zhaoqian $\mathrm{Liu}^{2,3}$ \\ 1. National Institution of Drug Clinical Trial, Xiangya Hospital, Central South University, Changsha, Hunan, P.R.China \\ 2. Department of Clinical Pharmacology, Xiangya Hospital, Central South University, Changsha, Hunan, P.R.China \\ 3. National Clinical Research Center for Geriatric Disorders, Xiangya Hospital, Central South University, Changsha, Hunan, P.R.China \\ 4. Department of Orthopaedics, The First Affiliated Hospital of the University of South China, Hengyang, Hunan, P.R.China \\ 5. Department of Otolaryngology Head and Neck Surgery, Xiangya Hospital, Central South University \\ 6. Otolaryngology Major Disease Research Key Laboratory of Hunan Province \\ 7. Changsha, Hunan, P.R.China. Department of pharmacy, Xiangya hospital, Central South University, Changsha, Hunan, P.R.China \\ *These authors contribute to this work equally
}

$\triangle$ Corresponding author: Professor Zhao-Qian Liu, Department of Clinical Pharmacology, Xiangya Hospital, Central South University, Changsha 410008; P. R. China; Institute of Clinical Pharmacology, Central South University, P. R. China. Tel: +86 731 84805380, Fax: +86 731 82354476, E-mail: zqliu@mail.csu.edu.cn.

(1) The author(s). This is an open access article distributed under the terms of the Creative Commons Attribution License (https://creativecommons.org/licenses/by/4.0/). See http://ivyspring.com/terms for full terms and conditions.

Received: 2019.05.12; Accepted: 2019.08.26; Published: 2019.11.01

\begin{abstract}
Adenylate cyclase 1 ( $A D C Y 1$ or $A C 1$ ) is a member of $A D C Y$ superfamily and was primarily found to be expressed in the brain. ADCY1 is responsible for catalyzing ATP to cyclic AMP (cAMP). As a secondary messenger, CAMP can regulate plenty of cellular activities. CAMP can perform its regulation in cellular transport through the binding to cAMP dependent protein kinases (PKAs), cAMP-activated guanine exchange factors (EPACs) and cyclic nucleotide-gated channels functioning in transduction of sensory signals (CNGs). Lung cancer is one of the leading factors of cancer-related death worldwide. Platinum-based chemotherapy is the first-line treatment for advanced lung cancer patients. In addition, surgical treatment, radiation treatment, and molecular targeted therapy are also therapeutic options for lung cancer patients in clinical settings. However, drug resistance and toxicity are the major obstacles that affect chemotherapy outcome and prognosis of lung cancer patients. And the therapeutic efficiency and adverse effects are varying with each individual. In recent years, investigations based on genetic sequencing have revealed the emerging role of $A D C Y 1$ mutations in affecting drug efficiency in various cancers such as lung cancer, esophageal cancer and colorectal cancer. The potential function of ADCY1 in chemotherapy resistance is of great importance to be noticed and investigated.
\end{abstract}

Key words: ADCY1, cAMP, Lung cancer, Drug resistance, Signaling pathway

\section{Introduction}

Lung cancer is one of the leading causes of cancer-related death worldwide in recent years [1]. There are two main kinds of lung cancer, including small-cell lung cancer (SCLC) and non-small-cell lung cancer (NSCLC). NSCLC is the most common subtype of lung cancer which accounts for approximately $85 \%$ [2]. However, most of the lung cancer patients were at advanced stages (stage III or IV) when diagnosed, which were not suitable for surgery. It is a huge challenge for treatment, with a 5-year survival rate less than $18 \%[3,4]$. Although there are multiple therapy strategies in treating lung cancer patients, such as surgery, chemotherapy, radiotherapy, molecular targeting treatment and immunotherapy [5-9], platinum-based chemotherapy is still the widely used first-line therapeutic regimen currently [10]. However, the dose related toxicity and drug resistance are the main obstacles limiting the therapeutic efficiency of platinum-based chemotherapy, which are vary greatly among 
individuals [11, 12]. More and more genetic polymorphisms have been identified to be associated with platinum-based chemotherapy toxicity and drug resistance [13], such as EGFR, eIF3a, ERCC1, WISP1, RAC1 [14-17]. All these shows that to find the potential biomarkers of drug resistance is of great importance.

Adenylate cyclase 1 (ADCY1 or AC1) is a member of ADCY superfamily and is primarily found to be expressed in the brain [18]. It is located in $7 \mathrm{p} 12.3$ and contains 22 exons; the molecular weight of its protein product is $130 \mathrm{kd}$. Current investigations of its function are mainly focused on brain, which found that ADCY1 is involved in various processes of the central nervous system [19]. ADCY1 was found to be highly expressed in inner ear cells comparing with the outer ear cells, and was also observed ubiquitously in the stereocilia on both the outer and inner hair cell of mouse. It could also affect hearing function in human and zebrafish [20]. ADCY1 was a main regulator of the cAMP signaling pathway [21]. It was also discovered to participate in a dopamine D4 receptors (D4Rs) modulated signaling pathway activated by neuronal PAS-domain protein 2 (NPAS2), which can regulate the circadian sensitivity of retinal ganglion cells [22]. Loss of ADCY1 function might lead to drastically-behavioral impairments in sensorimotor and social behaviors [23]. ADCY1 was also reported to be associated with alcohol addiction, and depression from adolescence to adult as well as in postpartum motherhood, which were accompanied by a down-regulation of ADCY1 expression [24, 25].

There are several report about the relationship between ADCY1 and drug resistance. ADCY1 was investigated to be significantly associated with the overall survival of patients with melanoma, and plays an important role in the metastases of melanoma [26]. What's more, ADCY1 was reported to be overexpressed in NSCLC tissues, which may related to the prognosis of NSCLC patients [27]. ADCY1 was hypermethylated in glioblastoma and associated with the survival time of the patients with malignant glioma [28]. It was also discovered to be high-expressed in multidrug-resistant cells, which means ADCY1 may related to the chemotherapy drug-resistance in esophageal carcinoma cells [29]. This review will be of great value to attract the public's attention to ADCY1 and its status as a novel biomarker in treating lung cancer patients. However, the specific mechanism underlying ADCY1 in regulating drug resistance in lung cancer patients is complex and unknown. DNA double-strand breaks (DSBs) pathway plays an important role in drug resistance, and DNA damage response (DDR) pathway participates in the cis-platinum induced cytotoxicity in non-small-cell lung cancer cells [30]. ADCY1 may regulate drug resistance mediated by its function in these processes widely recognized. For instance, ADCY1 was reported to be involved in DSBs in denuded oocytes, the mRNA expression of ADCY1 was up-regulated in these DSBs cumulus cells [31].

In this review, we summarized the recent advances of ADCY1 in lung cancer and its underlying mechanisms. First, we overviewed the relationship between ADCY1 and cAMP, as ADCY1 is a vital regulator which can catalyse ATP to cyclic adenosine 3', 5'-monophosphate (cAMP). cAMP is an important second messenger involved in diverse cellular responses including cell growth, cell differentiation, cell proliferation, apoptosis and metabolism. Then, we focused our eyes on the downstream effectors of cAMP signaling pathway adjusted by ADCY1, and their relationship with drug resistance in lung cancer. We also explored the three classical signaling pathways and other signalling pathways modified by cAMP in the regulation of drug resistance in lung cancer. Moreover, we summarized the probable lncRNAs modulated by cAMP which may contribute to drug resistance in lung cancer patients. Finally, we highlight the feasibility of ADCY1 as a potential biomarker to predict drug resistance, which may provide an important clue for future lung cancer treatments.

\section{The relationship between ADCY1 and cAMP}

ADCYs are activated by the stimulatory GTP-binding proteins ( $G$ proteins) which can response to various external signaling molecules through the regulation of $G$ protein-coupled receptors, such as neurotransmitters and hormones [32]. As a member of ADCYs, ADCY1 can catalyse ATP to cyclic adenosine $3^{\prime}, 5^{\prime}$-monophosphate (cAMP), and then the $3^{\prime}, 5^{\prime}$-cAMP will degrade into $5^{\prime}$-cAMP by cyclic nucleotide phosphodiesterases [33]. cAMP can perform its regulation in cellular transport through the binding to cAMP dependent protein kinases (PKAs), cAMP-activated guanine exchange factors (EPACs) and cyclic nucleotide-gated channels (CNGs) [34]. ADCY1 may affect DNA damage, cell apoptosis and cell proliferation through the regulation of the downstream effectors in cAMP signaling pathway (Figure 1).

PKAs and EPACs are the major targets of cAMP signaling pathway, activated PKAs-phosphorylatedenzymes can regulate relevant metabolism and transcription factors such as cAMP response element binding protein (CREB) [35]. These cAMP sensors can modulate diverse physiological processes either alone or in cooperation with PKAs. EPACs can regulate cell 
proliferation, differentiation, apoptosis, and inflammation; they also play a critical role in lung-related diseases [36]. Rap1 was identified as a main EPAC effector in several systems including lung [37]. The EPAC/Rap1 signaling is associated with the complex network of EMT signaling; whose dysfunction may cause lung cancer [38]. cAMP can activate CNGs to generate a depolarizing receptor potentially, it can also play an important role in the signaling of retinal photoreceptors and olfactory sensory neurons [39]. CNGs are composed of two CNGA2 subunits and two modulatory subunits (CNGA4 and CNGB1b). They are participating in ligand-gated activation and regulation of rapid termination of odorant signal in an olfactory sensory neuron through the coordination with each other [40]. Furthermore, PKAs, the main effector of cAMP, can participate in the regulation of cellular activities such as cell growth, proliferation, apoptosis and inflammation. EPACs can specifically activate the monomeric $G$ protein Rap [41]. CNGs are involved in the transduction of olfactory and visual signals. cAMP can perform its function in modulating diverse cellular activities through the regulation of these three main effectors (PKAs, EPACs and CNGs) [42].

ADCY1 can influence cAMP biosynthesis and degradation through the coupling by $\mathrm{Ca}^{2+}$. The interaction of ADCY1 with specific signaling proteins and regulatory factors can generate privileged domains in CAMP signaling pathways [43]. And the overexpression of ADCY1 is associated with the enhanced activity of extracellular signal-related kinase (ERK)/mitogen-activated protein kinase (MAPK) signaling pathway [44]. ADCY1 is a conversed element with a carboxyl tail, it can not only participate in the catalysis, but also is essential for membrane targeting due to its localization in the cytoplasm supporting cells. ADCY1 is engaged in abundant cellular signaling pathways and it can also contribute to physical behavior through the regulation of cAMP signaling pathway [45] (Figure 2).

\section{The association between cAMP and drug resistance in lung cancer}

The cAMP signaling pathway plays an essential role in diverse cellular responses including cell growth, cell differentiation, cell proliferation, apoptosis and metabolism. cAMP signaling can also modulates cancer cell death induced by anticancer drugs and $\gamma$-rays [46]. The normal regulation of DNA damage is of great significance, and its disordered process may lead to the cellular malignant transformation and tumor development. The DNA damage and repair mechanism is crucial for cell cycle progress $[47,48]$. Once the repair failed, the damaged cells are faced with cell death, including cell apoptosis. The abnormity of DNA damage and repair process can lead to cellular dysfunction and tumor development [49].

\section{The three classical signaling pathways modulated by cAMP to drug-resistance in lung cancer}

cAMP has been identified to participate in response to various DNA damaging statuses. It has been widely acknowledged that cAMP can regulate cell apoptosis by modulating the expression of Bcl-2 family proteins, the inhibitor of apoptosis proteins (IAPs) and the repair protein (XRCC1) of $\gamma$-ray-induced DNA damage in human lung cancer cells [50].

cAMP signaling can decrease the phosphorylation of Bcl-2 through the inhibition of JNK activation, which will result in the downregulation of cell autophagy and phosphorylation, and then reduce the ubiquitination and increase the expression of histone deacetylase 8 (HDAC8) [51]. As an anti-apoptosis family member, Bcl-2 can inhibit apoptosis by suppressing essential proapoptotic proteins with multiple Bcl-2 homology domains [52]. It can also modulate the permeabilization of the mitochondrial outer membrane (MOM), which is a critical step in apoptotic signal transduction, and then influence the cell survival of lung cancer cells. It has also been reported that the expression of Bcl-2 is associated with chemotherapy drug-resistance in malignant tumor patients. It has been reported that targeting Bcl-2 family proteins could overcome drug resistance in non-small cell lung cancer [53]. It means that cAMP may also affect chemotherapy drug-resistance through the regulation of Bcl-2.

As a novel inhibitor of apoptosis proteins that inhibit apoptosis triggered by a variety of stimuli, IAPs can play important roles in suppressing cell death and regulating DNA damage in the cell cycle. The translation initiation regulation of the internal ribosome entry sites is one of the mechanisms that can regulate the expression of IAP [54]. The cAMP-response-element-binding protein (CREB) can also regulate the activity of IAPs through the regulation of the enhancer sequence of IAPs to control cell apoptosis and DNA damage processes [55]. The overexpression of IAP2 could enhance chemotherapy resistance and promote cell survival in lung cancer cells [56]. 


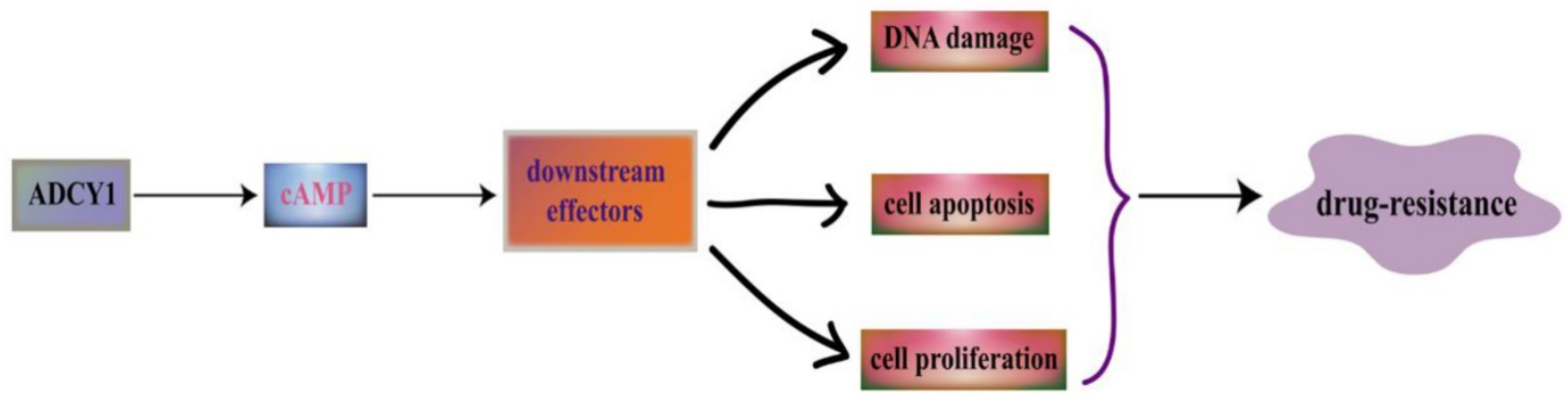

Figure 1. ADCY1 is a main regulator of the cAMP signaling pathway. ADCY1 can catalyse ATP to generate cyclic adenosine 3', 5'-monophosphate (cAMP), and then the 3', 5'-cAMP will degrade into 5'-cAMP by cyclic nucleotide phosphodiesterases. As a second messenger, cAMP participate in many important cellular activities. It can regulate its downstream effectors to have an influence on DNA damage, cell apoptosis and cell proliferation, which is linked with drug resistance.

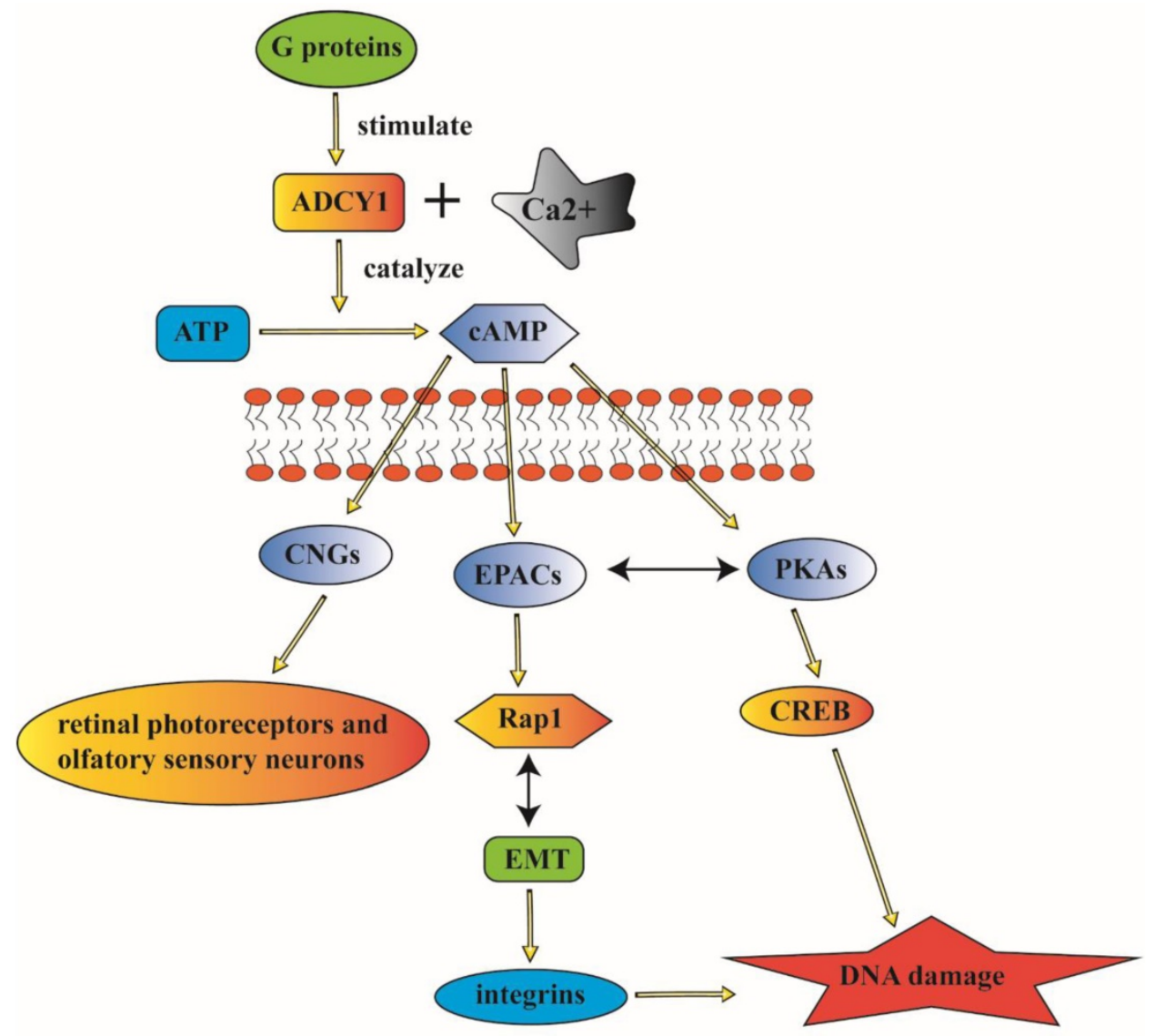

Figure 2. ADCY1 can participate in abundant cellular signaling pathways and contributes to physical behavior through the regulation of $c A M P$. ADCY1 can catalyze the formation of cAMP to modulate a series of signaling pathways. The major downstream effectors of cAMP including CNGs, EPACs and PKAs, which can regulate various cellular activities. And the mistaken control of ADCYl as the upstream modulator can contribute to complex disorder of these regulation. The complicated signaling pathway indicate the probable association between ADCYI and lung cancer.

The X-ray repair cross-complementing protein 1 (XRCC1) can affect cellular sensitivity to ionizing radiation. The expression and polymorphisms of $\mathrm{XRCC1}$ play an important role in DNA repair and it may be a prognosis biomarker for lung cancer patients treated with radiation or chemotherapy [57]. The cAMP signaling pathway can regulate DNA repair and hence contribute to radiation and chemotherapy resistance by inhibiting the expression of XRCC1in lung cancer patients [58]. The upstream signaling molecule of ADCY1 such as forskolin, an activator of ADCY1, can stimulate ADCY1 to catalyze
cAMP, can inhibit DNA damage, DNA repair and cell apoptosis which is caused by the degradation of XRCC1 in an Epac-dependent pathway in lung cancer cells [59].

In conclusion, cAMP can modulate DNA damage and repair to regulate cellular survival and the prognosis of lung cancer patients. It may regulate the sensitivity to radiation and chemotherapy in lung cancer patients through the modulation of these three popular effectors: Bcl-2, IAPs and XRCC1 [60] (Figure 3). 


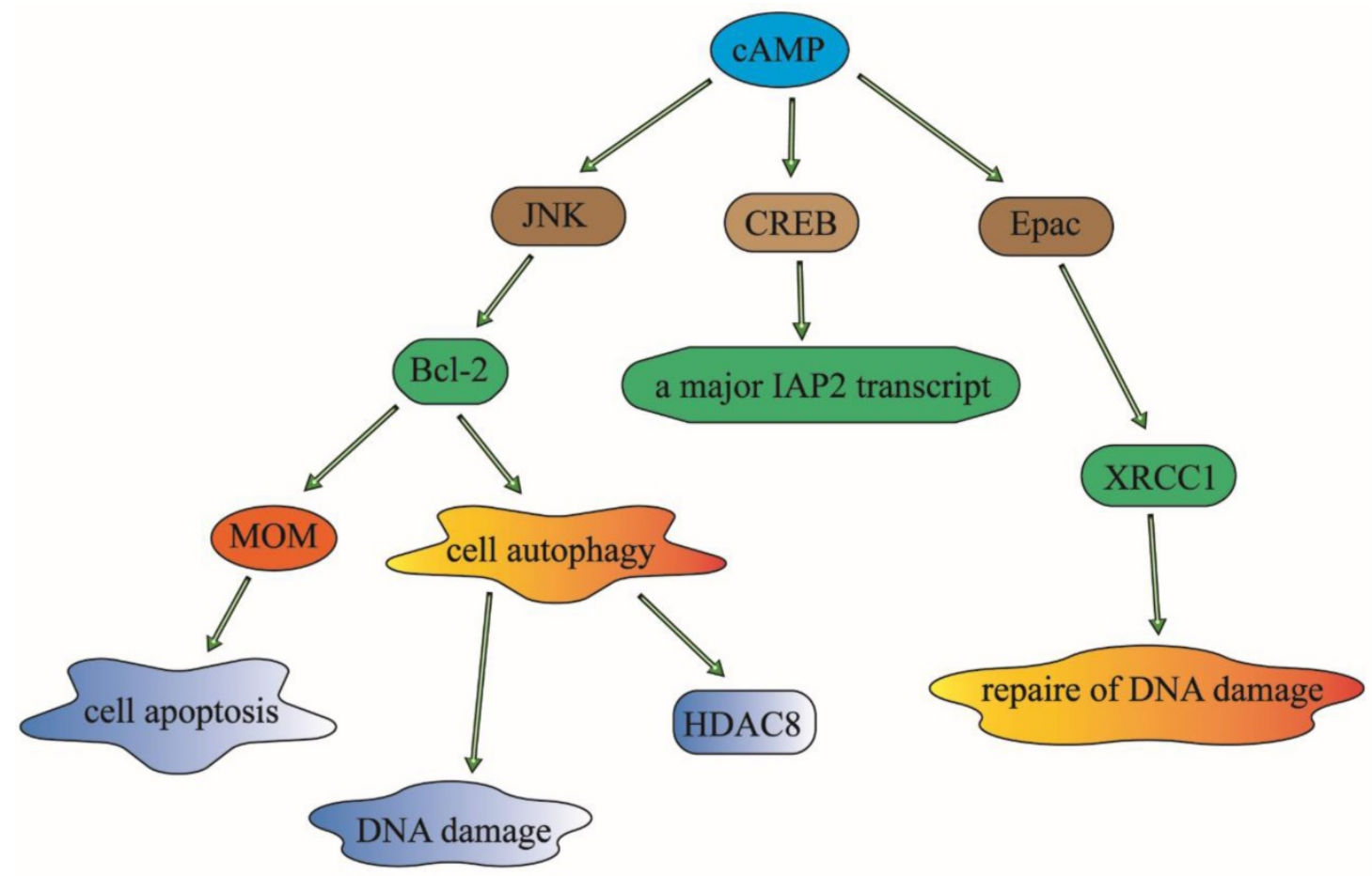

Figure 3. cAMP can modulate DNA damage, DNA repair and cell apoptosis to affect cellular survival or prognosis of patients with lung cancer. It can regulate the sensitivity to radiotherapy and chemotherapy in lung cancer patients, through the modulation of these three popular effectors: Bcl-2, IAPs and XRCC1. Given that these modulation of the three elements, cAMP can play an important role in the control of DNA damage and cell apoptosis. And then have an effect on the drug-resistance in lung cancer patients.

\section{Other signaling pathways contributing to drug resistance in lung cancer mediated by cAMP}

Investigations have also revealed that cAMP can regulate radiation-induced DNA damage by other effectors. The ataxia-telangiectasia mutated (ATM) protein kinase plays a vital role in harmonizing the cellular response to radiation-induced DNA damage [61]. cAMP can regulate DNA damage and enhance radiation-induced apoptosis in lung cancer cells through the inhibit of radiation-induced ATM activation by PKA-dependent activation of PP2A [62]. Sirtuin 6 (SIRT6) was detected to be expressed abnormally in NSCLC tissues; it can regulate genomic stability and cell viability via removing acetyl groups from histones [63]. cAMP signaling pathway can mediate the PKA-dependent inhibition of the Raf-MEK-ERK pathway to reduce the SIRT6 expression by promoting its ubiquitin-proteasomedependent degradation, and then facilitate radiationinduced apoptosis in lung cancer cells [64].

Multidrug-resistance (MDR) phenotypes are a major obstacle to the efficiency of cancer. The ATP-binding cassette transporter MDR1/Pglycoprotein (MDR1/P-gp), as an ABC transporter, has been identified to be associated with MDR in various cancers, such as lung cancer, ovarian cancer, laryngeal cancer [65]. There are some upstream modulators of CAMP participating in the regulation of MDR. As a $22 \mathrm{kDa} \mathrm{Ca}^{2+}$ binding protein, sorcin can induce the expression of MDR1/P-gp markedly through the activation of cAMP response element (CRE) located in the MDR1/p-gp promoter, and then regulate the CREB pathway which is associated with the MDR phenotype [66]. The expression of MDR1/p-gp was also found to be upregulated in doxorubicin-resistant non-small cell lung carcinoma (NCI-H460) cells. CD56 is a cell adhesion protein which is a member of the immunoglobulin superfamily. CD56 has been reported to be associated with chemotherapy resistance (such as EGFR-TKIs) in lung cancer patients [67]. cAMP can induce synaptophysin and chromogranin A, to increase the expression of CD56, which can make cells more sensitive to EGFR-TKIs (such as etoposide) and anthracycline compounds (such as DOX) by the regulation of cell adhesion [68].

MEK is activated in multiple cancer types such as non-small-cell lung cancer (NSCLC) and colorectal cancer (CRC). Selumetinib is a selective MEK1/2 inhibitor and is currently widely used in the treatment of NSCLC and SCLC [69]. It has been reported that treatment with 8-CL-cAMP, a selective PKA inhibitor (PKAI), can reverse MEK resistance in NSCLC cells through regulation of MAPK. This is in line with the experimental evidence that PKA is an upstream regulator in the MAPK and EGFR pathway [70]. There 
is also a functional link between EGFR/RAS-RAFMEK-ERK pathway and PKA expression and activity. The cAMP and the PKA activity were also found to be high-regulated in the taxol-resistant cells, which have been recognized to be associated with taxol resistance in human ovarian carcinoma [71]. OSU03013, a derivative of celecoxib, can compete with ATP to bind to cAMP-dependent protein kinase, and cause dephosphorylation of glycogen synthase kinase 3-beta (GSK3 $\beta)$, leading to beta-catenin ( $\beta$-catenin) degradation [72]. The interaction of GSK3 $\beta$ and $\beta$ catenin can regulate the invasion, proliferation and apoptosis of tumor cells, and modulate cisplatinbased chemotherapy sensitivity in lung cancer patients through the Wnt/ $\beta$-catenin signaling pathway [73].

Parathyroid hormone-related protein (PTHrP)(1-34) and PTHrP-(140-173) can protect lung cancer cells from apoptosis after the expose to radiation or chemotherapy drugs. A slight increase of cAMP was sufficient to protect lung cancer cells from apoptosis [74]. PKA activation was identified to induce resistance to cell apoptosis and regulate cell survival through PTHrP. The expression of the cystic fibrosis transmembrane conductance regulator (CFTR) gene has been reported to be related to multidrugresistance in lung cancer patients [75]. It can increase in short-circuit current (Isc) in response to mediators through the cAMP-dependent $\mathrm{Cl}$ - secretion, which can mediate the activity of CFTR to modulate multidrug-resistance in lung cancer [76]. There is an investigation found that the cyclic adenosine 3',5'-monophosphate (cAMP) analogue, 8-chlorocAMP (8-Cl-cAMP), had a collateral growthinhibitory effect on cis-diammine dichloroplatinum (CDDP) resistant cells, which means that 8-Cl-cAMP may be as a useful tool as cAMP to predict cis-diammine dichloroplatinum (CDDP) sensitivity [77]. 8-Cl-cAMP has been shown to selectively bind to the site I receptor of the type II regulatory subunit (RII) of cAMP-dependent protein kinase, which means it is correlated with CDDP sensitivity in lung cancer [78].

In conclusion, cAMP or its analogue (8-ClcAMP) can regulate multidrug-resistance in lung cancer patients. There are plenty of downstream effectors participating in the cAMP signaling pathway such as the classical PKA and CREB. These complex but well-ordered signaling pathways can regulate the majority of cellular activities such as cell apoptosis, cell growth, cell cycle, cell proliferation, which may have an effect on multidrug-resistance in the treatment of lung cancer patients [79] (Figure 4).

\section{The association between cAMP and lncRNA to drug resistance in lung cancer}

Long noncoding RNAs (lncRNAs) play a diverse range of biological roles, including regulation of gene expression. In recent years, lncRNAs have been recognized as emerging regulators in tumorigenesis [80]. LncRNAs have been also identified to be involved in drug resistance, and hence found as potential biomarkers to predict prognosis of various cancers such as esophageal cancer, gastric cancer and lung cancer [81].

As a second messenger in multi-cellular activities, cAMP can regulate various lncRNAs effectors. COX-2 is an anticancer target; however, the drug resistance of COX-2 inhibitors is a major obstacle limiting their therapy efficiency in clinical treatment [82]. CAMP can regulate the glycosylated COX-2 and PGE-2 to affect CRTC1 activation and LKB1 status, which means that cAMP can play an important role in the sensitivity to COX-2 inhibitors through the CRTC1 signaling pathway [83]. LKB1 is a tumor suppressor gene which is frequently mutated and inactivated in NSCLC. Loss of LKB1 will promote cancer progression and influence therapeutic responses in preclinical investigations, due to its effect on drug efficacy through the regulation of MDR1 expression [84]. cAMP can regulate LKB1 through cAMP-responsive element-binding protein (CREB) to affect the LncRNA LNC00473's activity, and then play an vital role in multi-drug resistance of lung cancer patients [85], given that overexpressed LINC00473 is correlated with poor prognosis, and stabilized expression of LINC00473 was required for cell proliferation and cell survival in LKB1-inactivated NSCLC cells. LNC00473 can also interact with cAMP/CREB through NONO, which is an element in cAMP signaling pathway [86]. cAMP can also regulate LINC00473 to mediate decidualization of human endometrial stromal cells, which is through the cAMP-PKA pathway to modulate the phosphorylation of STAT3. It means that CAMP-PKA or CAMP-CREB can regulate the activity of LINC00473 through different pathways.

There are many LncRNAs highly up-regulated in liver cancer (HULC) known as Lnc HULC. They play important roles in tumorigenesis and metastasis. And they can be act as biomarkers to predict drug efficacy and prognosis of various cancers [87]. cAMP can regulate Lnc HULC through a CREB binding site, and then modulate PRKACB activity through targeting the miR-372. CAMP can regulate tumorigenesis, cell survival and modulate multi-drug resistance in liver cancer through this signaling pathway [88]. MEG3 is a long non-coding RNA which 
is a human maternally expressed gene associated with tumorigenesis and cell proliferation. Alteration of the expression of MEG3 is also correlated with cis-platinum resistance in lung adenocarcinoma. MEG3 can mediate chemosensitivity through the $\mathrm{WNT} / \beta$-catenin signaling pathway by the regulation of p53, $\beta$-catenin and survivin [89]. cAMP can regulate the activity of MEG3 through a cAMP response element (CRE) in the proximal promoter region of MEG3, and then mediate cis-platinum resistance in lung cancer patients through regulating interactions between MEG3 and its downstream effectors [90].

In summary, cAMP can regulate multidrug resistance in lung cancer and other malignant tumors through the regulation of particular lncRNAs involving in different signaling pathways, which is of great value in clinical treatment for lung cancer therapy (Figure 5).

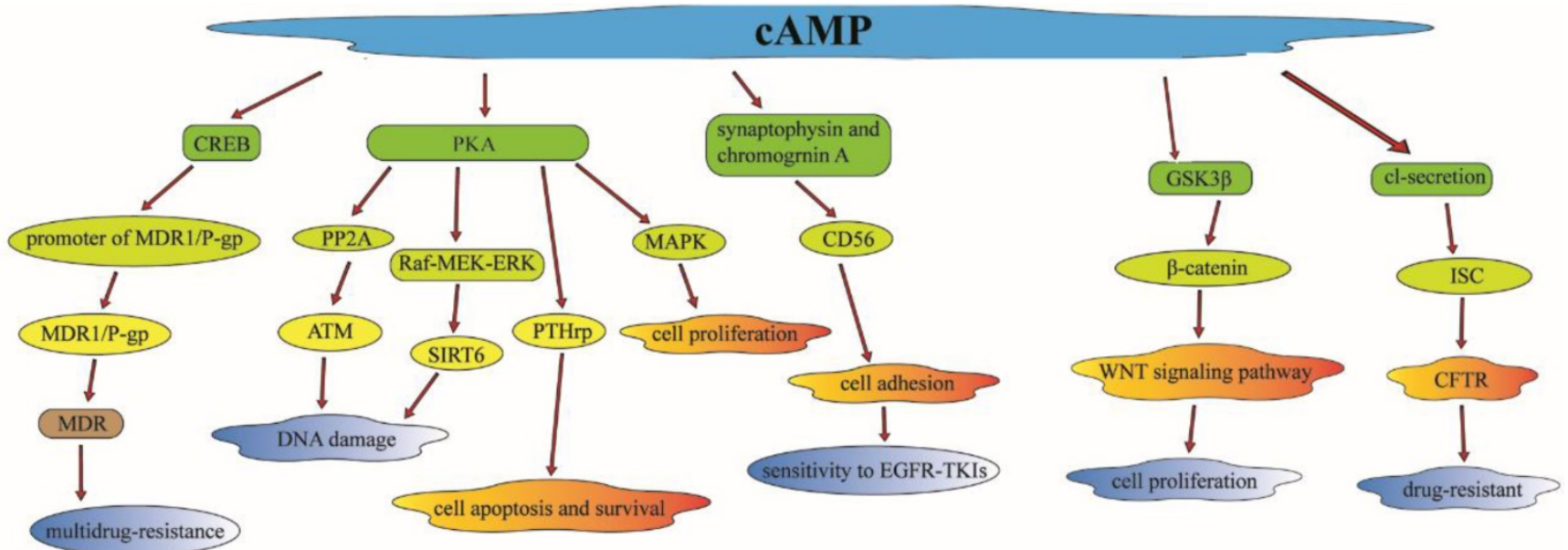

Figure 4. cAMP can regulate multidrug-resistance in lung cancer through the regulation of plenty of downstream effectors, which is participating in the cAMP signaling pathway such as the classical PKA and CREB or some other useful effectors. Through the regulation of these downstream effectors, cAMP can modulate cell apoptosis, cell survival, cell growth, cell cycle and cell proliferation which will have an effect on multidrug-resistance in lung cancer treatment.

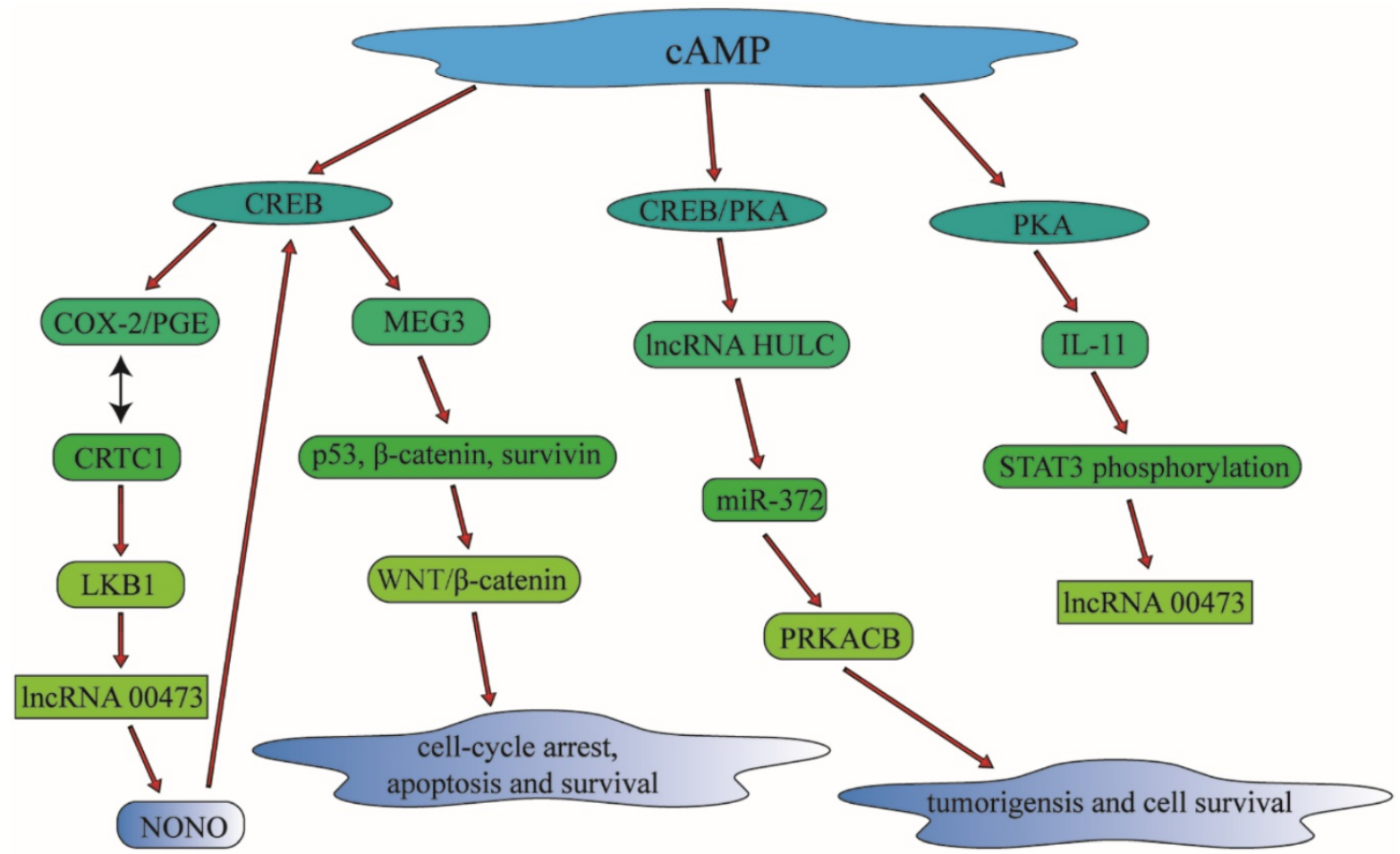

Figure 5. cAMP can control multidrug resistance in lung cancer and other malignant tumors through the regulation of particular IncRNAs involving in different signaling pathways, which is of great value in clinical treatment for lung cancer therapy. CAMP can modulate CREB and PKA to have an influence in the IncRNA such as IncRNA HULC and IncRNA 00473. Through the regulation of IncRNA, cAMP can also make a connection with cell apoptosis and cell survival, which is associated with drug-resistance significantly. 


\section{Conclusion}

Here we reviewed for the first time of ADCY1 and its association with drug resistance in lung cancer. As a member of adenylate cyclase (ADCY), ADCY1 was first found to be expressed abundantly in brain and subsequent studies was mainly focused on its role in brain diseases. Mutations of ADCY1 were revealed to be associated with hearing impairment or other disorders in the nervous system. In this review, we focus on the function of ADCY1 in the regulation of multidrug resistance in lung cancer mediated by cAMP, which is a second messenger participating in plenty of cellular activities. cAMP has also been reported to be involved in the regulation of drug resistance in lung cancer through the downstream effectors in different signaling pathways such as the effectors belonging to DNA damage signaling pathway and specific lncRNAs. Taken together, ADCY1 is of great significance to be a novel biomarker to predict drug resistance in lung cancer patients. However, the specific mechanism of ADCY1 regulating drug resistance need to be further investigated.

\section{Abbreviations}

EGFR: epidermal growth factor receptor; eIF3a: Eukaryotic translation initiation factor 3 subunit A; ERCC1: ERCC excision repair 1; WISP1: WNT1 inducible signaling pathway protein 1; RAC1: ras-related C3 botulinum toxin substrate.

\section{Acknowledgements}

This work was supported by the National High-tech R\&D Program of China (863 Program) (2012AA02A517), National Natural Science Foundation of China (81373490, 81573508), Hunan Provincial Science and Technology Plan of China (2015TP1043), Hunan Provincial Natural Science Foundation of China (2019JJ50946), and Youth Science Foundation of Xiangya Hospital, Central South University (2018Q014).

\section{Open Access}

This article is distributed under the terms of the Creative Commons Attribution 4.0 International License (http://creativecommons.org/licenses/by/ $4.0 /$ ), which permits unrestricted use, distribution, and reproduction in any medium, provided you give appropriate credit to the original author(s) and the source, provide a link to the Creative Commons license, and indicate if changes were made.

\section{Competing Interests}

The authors have declared that no competing interest exists.

\section{References}

1. Siegel RL, Miller KD, Jemal A. Cancer statistics, 2015. CA: a cancer journal for clinicians. 2015; 65: 5-29.

2. Siegel RL, Miller KD, Jemal A. Cancer statistics, 2016. CA: a cancer journal for clinicians. 2016; 66: 7-30.

3. Kuribayashi K, Funaguchi N, Nakano T. Chemotherapy for advanced non-small cell lung cancer with a focus on squamous cell carcinoma. Journal of cancer research and therapeutics. 2016; 12: 528-34.

4. Chen W, Zheng R, Baade PD, Zhang S, Zeng H, Bray F, et al. Cancer statistics in China, 2015. CA: a cancer journal for clinicians. 2016; 66: 115-32.

5. Paleiron N, Bylicki O, Andre M, Riviere E, Grassin F, Robinet G, et al. Targeted therapy for localized non-small-cell lung cancer: a review. OncoTargets and therapy. 2016; 9: 4099-104.

6. Khanna P, Blais N, Gaudreau PO, Corrales-Rodriguez L. Immunotherapy Comes of Age in Lung Cancer. Clinical lung cancer. 2016.

7. Hellmann MD, Li BT, Chaft JE, Kris MG. Chemotherapy remains an essential element of personalized care for persons with lung cancers. Annals of oncology : official journal of the European Society for Medical Oncology / ESMO. 2016

8. Lanuti M. Surgical Management of Oligometastatic Non-Small Cell Lung Cancer. Thoracic surgery clinics. 2016; 26: 287-94

9. Timmerman RD, Bizekis CS, Pass HI, Fong Y, Dupuy DE, Dawson LA, et al. Local surgical, ablative, and radiation treatment of metastases. CA: a cancer journal for clinicians. 2009; 59: 145-70.

10. Group NM-aC. Preoperative chemotherapy for non-small-cell lung cancer: a systematic review and meta-analysis of individual participant data. Lancet. 2014; 383: 1561-71.

11. Chong CR, Janne PA. The quest to overcome resistance to EGFR-targeted therapies in cancer. Nature medicine. 2013; 19: 1389-400.

12. Zheng Y, Deng Z, Yin J, Wang S, Lu D, Wen X, et al. The association of genetic variations in DNA repair pathways with severe toxicities in NSCLC patients undergoing platinum-based chemotherapy. International journal of cancer. 2017; 141: 2336-47.

13. O'Grady S, Finn SP, Cuffe S, Richard DJ, O'Byrne KJ, Barr MP. The role of DNA repair pathways in cisplatin resistant lung cancer. Cancer treatment reviews. 2014; 40: 1161-70.

14. Yin JY, Meng XG, Qian CY, Li XP, Chen J, Zheng Y, et al. Association of positively selected eIF3a polymorphisms with toxicity of platinum-based chemotherapy in NSCLC patients. Acta pharmacologica Sinica. 2015; 36: $375-84$.

15. Chen J, Yin J, Li X, Wang Y, Zheng Y, Qian C, et al. WISP1 polymorphisms contribute to platinum-based chemotherapy toxicity in lung cancer patients. International journal of molecular sciences. 2014; 15: 21011-27.

16. Zou T, Yin J, Zheng W, Xiao L, Tan L, Chen J, et al. Rho GTPases: RAC1 polymorphisms affected platinum-based chemotherapy toxicity in lung cancer patients. Cancer chemotherapy and pharmacology. 2016; 78: 249-58.

17. Yin JY, Zhang JT, Zhang W, Zhou HH, Liu ZQ. eIF3a: A new anticancer drug target in the eIF family. Cancer letters. 2018; 412: 81-7.

18. Abdel-Majid RM, Leong WL, Schalkwyk LC, Smallman DS, Wong ST, Storm DR, et al. Loss of adenylyl cyclase I activity disrupts patterning of mouse somatosensory cortex. Nature genetics. 1998; 19: 289-91.

19. Wang H, Storm DR. Calmodulin-regulated adenylyl cyclases: cross-talk and plasticity in the central nervous system. Molecular pharmacology. 2003; 63: 463-8

20. Santos-Cortez RL, Lee K, Giese AP, Ansar M, Amin-Ud-Din M, Rehn K, et al. Adenylate cyclase 1 (ADCY1) mutations cause recessive hearing impairment in humans and defects in hair cell function and hearing in zebrafish. Human molecular genetics. 2014; 23: 3289-98.

21. Arakawa H, Akkentli F, Erzurumlu RS. Region-Specific Disruption of Adenylate Cyclase Type 1 Gene Differentially Affects Somatosensorimotor Behaviors in Mice. eNeuro. 2014; 1.

22. Hwang CK, Chaurasia SS, Jackson CR, Chan GC, Storm DR, Iuvone PM. Circadian rhythm of contrast sensitivity is regulated by a dopamine-neuronal PAS-domain protein 2-adenylyl cyclase 1 signaling pathway in retinal ganglion cells. The Journal of neuroscience : the official journal of the Society for Neuroscience. 2013; 33: 14989-97.

23. Arakawa H, Akkentli F, Erzurumlu RS. Region-Specific Disruption of Adenylate Cyclase Type 1 Gene Differentially Affects Somatosensorimotor Behaviors in Mice $(1,2,3)$. eNeuro. 2014; 1.

24. Zou $\mathrm{H}$, Wang $\mathrm{K}$, Gao $\mathrm{Y}$, Song $\mathrm{H}$, Xie $\mathrm{O}$, Jin $\mathrm{M}$, et al. Chronic alcohol consumption from adolescence-to-adulthood in mice--hypothalamic gene expression changes in the dilated cardiomyopathy signaling pathway. BMC neuroscience. 2014; 15 : 61.

25. Zhao C, Eisinger BE, Driessen TM, Gammie SC. Addiction and reward-related genes show altered expression in the postpartum nucleus accumbens. Frontiers in behavioral neuroscience $2014 ; 8: 388$. 
26. Chen J, Wu F, Shi Y, Yang D, Xu M, Lai Y, et al. Identification of key candidate genes involved in melanoma metastasis. Molecular medicine reports. 2019.

27. He RQ, Li XJ, Liang L, Xie Y, Luo DZ, Ma J, et al. The suppressive role of miR-542-5p in NSCLC: the evidence from clinical data and in vivo validation using a chick chorioallantoic membrane model. BMC cancer. 2017; 17: 655.

28. Ma J, Hou X, Li M, Ren H, Fang S, Wang X, et al. Genome-wide methylation profiling reveals new biomarkers for prognosis prediction of glioblastoma. Journal of cancer research and therapeutics. 2015; 11 Suppl 2: C212-5.

29. Yang LX, Li BL, Liu XH, Yuan Y, Lu CJ, Chen R, et al. RNA-seq reveals determinants of sensitivity to chemotherapy drugs in esophageal carcinoma cells. International journal of clinical and experimental pathology. 2014; 7 : 1524-33.

30. Sears CR, Cooney SA, Chin-Sinex H, Mendonca MS, Turchi JJ. DNA damage response (DDR) pathway engagement in cisplatin radiosensitization of non-small cell lung cancer. DNA repair. 2016; 40: 35-46.

31. Sun MH, Zheng J, Xie FY, Shen W, Yin S, Ma JY. Cumulus Cells Block Oocyte Meiotic Resumption via Gap Junctions in Cumulus Oocyte Complexes Subjected to DNA Double-Strand Breaks. PloS one. 2015; 10: e0143223.

32. Rosenbaum DM, Rasmussen SG, Kobilka BK. The structure and function of G-protein-coupled receptors. Nature. 2009; 459: 356-63.

33. Zhang F, Zhang L, Qi Y, Xu H. Mitochondrial cAMP signaling. Cellular and molecular life sciences : CMLS. 2016.

34. Lewis AE, Aesoy R, Bakke M. Role of EPAC in cAMP-Mediated Actions in Adrenocortical Cells. Frontiers in endocrinology. 2016; 7: 63.

35. Bos JL. Epac: a new cAMP target and new avenues in cAMP research. Nature reviews Molecular cell biology. 2003; 4: 733-8.

36. Mansilla Pareja ME, Gauron MC, Robledo E, Aguilera MO, Colombo MI. The cAMP effectors, Rap2b and EPAC, are involved in the regulation of the development of the Coxiella burnetii containing vacuole by altering the fusogenic capacity of the vacuole. PloS one. 2019; 14: e0212202.

37. Roscioni SS, Elzinga CR, Schmidt M. Epac: effectors and biological functions. Naunyn-Schmiedeberg's archives of pharmacology. 2008; 377: 345-57.

38. Balzac F, Avolio M, Degani S, Kaverina I, Torti M, Silengo L, et al. E-cadherin endocytosis regulates the activity of Rap1: a traffic light GTPase at the crossroads between cadherin and integrin function. Journal of cell science. 2005; 118: 4765-83.

39. Kaupp UB, Seifert R. Cyclic nucleotide-gated ion channels. Physiological reviews. 2002; 82: 769-824.

40. Pifferi S, Boccaccio A, Menini A. Cyclic nucleotide-gated ion channels in sensory transduction. FEBS letters. 2006; 580: 2853-9.

41. Di Giacomo M, Camaioni A, Klinger FG, Bonfiglio R, Salustri A. Cyclic AMP-elevating Agents Promote Cumulus Cell Survival and Hyaluronan Matrix Stability, Thereby Prolonging the Time of Mouse Oocyte Fertilizability. The Journal of biological chemistry. 2016; 291: 3821-36.

42. Gao M, Ma Y, Bast RC, Jr., Li Y, Wan L, Liu Y, et al. Epac1 knockdown inhibits the proliferation of ovarian cancer cells by inactivating AKT/Cyclin D1/CDK4 pathway in vitro and in vivo. Medical oncology. 2016; 33: 73 .

43. Tasken K, Aandahl EM. Localized effects of cAMP mediated by distinct routes of protein kinase A. Physiological reviews. 2004; 84: 137-67.

44. Seino S, Shibasaki T. PKA-dependent and PKA-independent pathways for cAMP-regulated exocytosis. Physiological reviews. 2005; 85: 1303-42.

45. Zachariou V, Liu R, LaPlant Q, Xiao G, Renthal W, Chan GC, et al. Distinct roles of adenylyl cyclases 1 and 8 in opiate dependence: behavioral, electrophysiological, and molecular studies. Biological psychiatry. 2008; 63: 1013-21.

46. Spina A, Di Maiolo F, Esposito A, D'Auria R, Di Gesto D, Chiosi E, et al. Integrating leptin and cAMP signalling pathways in triple-negative breast cancer cells. Frontiers in bioscience. 2013; 18: 133-44.

47. Li ZH, Wang YZ, Liu J, Zhu M, Du JB, Yuan J, et al. [DNA damage associated genetic variants contribute to lung cancer susceptibility in a Han Chinese population]. Zhonghua yu fang yi xue za zhi [Chinese journal of preventive medicine]. 2016; 50: 721-7.

48. Peng F, Liu Y, He C, Kong Y, Ouyang Q, Xie X, et al. Prediction of platinum-based chemotherapy efficacy in lung cancer based on LC-MS metabolomics approach. Journal of pharmaceutical and biomedical analysis. 2018; 154: 95-101.

49. Song EA, Kim H. Docosahexaenoic Acid Induces Oxidative DNA Damage and Apoptosis, and Enhances the Chemosensitivity of Cancer Cells. International journal of molecular sciences. 2016; 17 .

50. Cho EA, Juhnn YS. The cAMP signaling system inhibits the repair of gamma-ray-induced DNA damage by promoting Epac1-mediated proteasomal degradation of XRCC1 protein in human lung cancer cells. Biochemical and biophysical research communications. 2012; 422: 256-62.

51. Park JY, Juhnn YS. cAMP signaling increases histone deacetylase 8 expression by inhibiting JNK-dependent degradation via autophagy and the proteasome system in H1299 lung cancer cells. Biochemical and biophysical research communications. 2016; 470: 336-42.

52. Willis SN, Fletcher JI, Kaufmann T, van Delft MF, Chen L, Czabotar PE, et al. Apoptosis initiated when $\mathrm{BH} 3$ ligands engage multiple $\mathrm{Bcl}-2$ homologs, not Bax or Bak. Science. 2007; 315: 856-9.

53. Wesarg E, Hoffarth S, Wiewrodt R, Kroll M, Biesterfeld S, Huber C, et al. Targeting BCL-2 family proteins to overcome drug resistance in non-small cell lung cancer. International journal of cancer. 2007; 121: 2387-94.

54. Dong Z, Nishiyama J, Yi X, Venkatachalam MA, Denton M, Gu S, et al. Gene promoter of apoptosis inhibitory protein IAP2: identification of enhancer elements and activation by severe hypoxia. The Biochemical journal. 2002; 364: 413-21.

55. Wong JC, Bathina M, Fiscus RR. Cyclic GMP/protein kinase G type-Ialpha (PKG-Ialpha) signaling pathway promotes CREB phosphorylation and maintains higher c-IAP1, livin, survivin, and Mcl-1 expression and the inhibition of PKG-Ialpha kinase activity synergizes with cisplatin in non-small cell lung cancer cells. Journal of cellular biochemistry. 2012; 113: 3587-98.

56. Jeon YK, Kim CK, Koh J, Chung DH, Ha GH. Pellino-1 confers chemoresistance in lung cancer cells by upregulating cIAP2 through Lys63-mediated polyubiquitination. Oncotarget. 2016.

57. Jeon YK, Kim CK, Koh J, Chung DH, Ha GH. Pellino-1 confers chemoresistance in lung cancer cells by upregulating cIAP2 through Lys63-mediated polyubiquitination. Oncotarget. 2016; 7: 41811-24.

58. Zhai XH, Huang J, Wu FX, Zhu DY, Wang AC. Impact of XRCC1, GSTP1, and GSTM1 Polymorphisms on the Survival of Ovarian Carcinoma Patients Treated with Chemotherapy. Oncology research and treatment. 2016; 39: 440-6.

59. Ginsberg G, Angle K, Guyton K, Sonawane B. Polymorphism in the DNA repair enzyme XRCC1: utility of current database and implications for human health risk assessment. Mutation research. 2011; 727: 1-15.

60. Levenson VV, Davidovich IA, Roninson IB. Pleiotropic resistance to DNA-interactive drugs is associated with increased expression of genes involved in DNA replication, repair, and stress response. Cancer research. 2000; 60: 5027-30.

61. Lim JA, Juhnn YS. Isoproterenol increases histone deacetylase 6 expression and cell migration by inhibiting ERK signaling via PKA and Epac pathways in human lung cancer cells. Experimental \& molecular medicine. 2016; 48: e204.

62. Bakkenist CI, Kastan MB. DNA damage activates ATM through intermolecular autophosphorylation and dimer dissociation. Nature. 2003; 421: 499-506.

63. Cho EA, Kim EJ, Kwak SJ, Juhnn YS. cAMP signaling inhibits radiation-induced ATM phosphorylation leading to the augmentation of apoptosis in human lung cancer cells. Molecular cancer. 2014; 13: 36.

64. Lu TP, Tsai MH, Lee JM, Hsu CP, Chen PC, Lin CW, et al. Identification of a novel biomarker, SEMA5A, for non-small cell lung carcinoma in nonsmoking women. Cancer epidemiology, biomarkers \& prevention : a publication of the American Association for Cancer Research, cosponsored by the American Society of Preventive Oncology. 2010; 19: 2590-7.

65. Kim EJ, Juhnn YS. Cyclic AMP signaling reduces sirtuin 6 expression in non-small cell lung cancer cells by promoting ubiquitin-proteasomal degradation via inhibition of the Raf-MEK-ERK (Raf/mitogen-activated extracellular signal-regulated kinase/extracellular signal-regulated kinase) pathway. The Journal of biological chemistry. 2015; 290: 9604-13.

66. Surowiak P, Pawelczyk K, Maciejczyk A, Pudelko M, Kolodziej J, Zabel M, et al. Positive correlation between cyclooxygenase 2 and the expression of $\mathrm{ABC}$ transporters in non-small cell lung cancer. Anticancer research. 2008; 28: 2967-74.

67. Yamagishi N, Nakao R, Kondo R, Nishitsuji M, Saito Y, Kuga T, et al. Increased expression of sorcin is associated with multidrug resistance in leukemia cells via up-regulation of MDR1 expression through cAMP response element-binding protein. Biochemical and biophysical research communications. 2014; 448: 430-6

68. Han L, Wang YF, Zhang Y, Wang N, Guo XJ, Yang JK, et al. Increased expression and function of P-glycoprotein in peripheral blood CD56+ cells is associated with the chemoresistance of non-small-cell lung cancer. Cancer chemotherapy and pharmacology. 2012; 70: 365-72.

69. Chang Y, Kim SY, Choi YJ, So KS, Rho JK, Kim WS, et al. Neuroendocrine differentiation in acquired resistance to epidermal growth factor receptor tyrosine kinase inhibitor. Tuberculosis and respiratory diseases. 2013; 75: 95-103.

70. Bernabe R, Patrao A, Carter L, Blackhall F, Dean E. Selumetinib in the treatment of non-small-cell lung cancer. Future oncology. 2016; 12: 2545-60.

71. Kim JY, Welsh EA, Fang B, Bai Y, Kinose F, Eschrich SA, et al. Phosphoproteomics Reveals MAPK Inhibitors Enhance MET- and EGFR-Driven AKT Signaling in KRAS-Mutant Lung Cancer. Molecular cancer research : MCR. 2016; 14: 1019-29.

72. Parekh HK, Adikari M, Vennapusa B. Differential partitioning of Galphai1 with the cellular microtubules: a possible mechanism of development of Taxol resistance in human ovarian carcinoma cells. Journal of molecular signaling. 2006; $1: 3$.

73. Tan YH, Lee KH, Lin T, Sun YC, Hsieh-Li HM, Juan HF, et al. Cytotoxicity and proteomics analyses of OSU03013 in lung cancer. Clinical cancer research : an official journal of the American Association for Cancer Research. 2008; 14: 1823-30.

74. Luo K, Gu X, Liu J, Zeng G, Peng L, Huang H, et al. Inhibition of disheveled-2 resensitizes cisplatin-resistant lung cancer cells through down-regulating Wnt/beta-catenin signaling. Experimental cell research. 2016; 347: 105-13.

75. Kobayashi N, Rosenthal ER, Yoshimura K, Crystal RG. Transfer of a constitutive viral promoter-cystic fibrosis transmembrane conductance regulator cDNA to human epithelial cells conveys resistance to down-regulation of cAMP-regulated $\mathrm{Cl}$ - secretion in the presence of inflammatory stimuli. Nucleic acids research. 1994; 22: 4470-6.

76. Shen BQ, Finkbeiner WE, Wine JJ, Mrsny RJ, Widdicombe JH. Calu-3: a human airway epithelial cell line that shows cAMP-dependent $\mathrm{Cl}$ - secretion. The American journal of physiology. 1994; 266: L493-501. 
77. Nishio K, Morikage T, Kubota N, Ohmori T, Takeda Y, Fujiwara Y, et al. Alteration of type II regulatory subunit of cAMP-dependent protein kinase in human cisplatin-resistant cells as a basis of collateral sensitivity to 8-chloro-cAMP. Japanese journal of cancer research : Gann. 1992; 83: 754-60.

78. Hussain M, Tang F, Liu J, Zhang J, Javeed A. Dichotomous role of protein kinase A type I (PKAI) in the tumor microenvironment: a potential target for 'two-in-one' cancer chemoimmunotherapeutics. Cancer letters. 2015; 369: 9-19.

79. Gong S, Chen Y, Meng F, Zhang Y, Li C, Zhang G, et al. Roflumilast enhances cisplatin-sensitivity and reverses cisplatin-resistance of ovarian cancer cells via cAMP/PKA/CREB-FtMt signalling axis. Cell proliferation. 2018; 51: e12474.

80. Wang L, Sun Y, Yi J, Wang X, Liang J, Pan Z, et al. Targeting H19 by lentivirus-mediated RNA interference increases A549 cell migration and invasion. Experimental lung research. 2016: 1-8.

81. Chen S, Wu H, Lv N, Wang H, Wang Y, Tang Q, et al. LncRNA CCAT2 predicts poor prognosis and regulates growth and metastasis in small cell lung cancer. Biomedicine \& pharmacotherapy = Biomedecine \& pharmacotherapie. 2016; 82: 583-8.

82. Said-Elbahr R, Nasr M, Alhnan MA, Taha I, Sammour O. Nebulizable colloidal nanoparticles co-encapsulating a COX-2 inhibitor and a herbal compound for treatment of lung cancer. European journal of pharmaceutics and biopharmaceutics : official journal of Arbeitsgemeinschaft fur Pharmazeutische Verfahrenstechnik eV. 2016; 103: 1-12.

83. Cao $\mathrm{C}$, Gao $\mathrm{R}$, Zhang $\mathrm{M}$, Amelio $\mathrm{AL}$, Fallahi $\mathrm{M}$, Chen $\mathrm{Z}$, et al. Role of LKB1-CRTC1 on glycosylated COX-2 and response to COX-2 inhibition in lung cancer. Journal of the National Cancer Institute. 2015; 107: 358.

84. Mao K, Liu F, Liu X, Khuri FR, Marcus AI, Li M, et al. Re-expression of LKB1 in LKB1-mutant EKVX cells leads to resistance to paclitaxel through the up-regulation of MDR1 expression. Lung cancer. 2015; 88: 131-8.

85. Chen $\mathrm{Z}, \mathrm{Li}$ JL, Lin S, Cao C, Gimbrone NT, Yang R, et al. cAMP/CREB-regulated LINC00473 marks LKB1-inactivated lung cancer and mediates tumor growth. The Journal of clinical investigation. 2016; 126: 2267-79.

86. Ong SA, Tan JJ, Tew WL, Chen KS. Rasd1 modulates the coactivator function of NonO in the cyclic AMP pathway. PloS one. 2011; 6: e24401.

87. $\mathrm{Li} \mathrm{CH}$, Chen Y. Targeting long non-coding RNAs in cancers: progress and prospects. The international journal of biochemistry \& cell biology. 2013; 45: 1895-910.

88. Wang J, Liu X, Wu H, Ni P, Gu Z, Qiao Y, et al. CREB up-regulates long non-coding RNA, HULC expression through interaction with microRNA-372 in liver cancer. Nucleic acids research. 2010; 38: 5366-83.

89. Xia Y, He Z, Liu B, Wang P, Chen Y. Downregulation of Meg3 enhances cisplatin resistance of lung cancer cells through activation of the WNT/beta-catenin signaling pathway. Molecular medicine reports. 2015; 12: 4530-7.

90. Zhao J, Zhang X, Zhou Y, Ansell PJ, Klibanski A. Cyclic AMP stimulates MEG3 gene expression in cells through a cAMP-response element (CRE) in the MEG3 proximal promoter region. The international journal of biochemistry \& cell biology. 2006; 38: 1808-20. 